\title{
A DC Power Flow Extension
}

\author{
Theodoros Kyriakidis, Rachid Cherkaoui, Maher Kayal \\ Electronics Laboratory \& Power System Group \\ École Polytechnique Fédérale de Lausanne \\ CH-1015 Lausanne, Switzerland \\ theodoros.kyriakidis@epfl.ch
}

\begin{abstract}
In this work an extension of the well-known DC power flow method is presented. A normal DC power flow of the system is executed to determine voltage angles and a novel derivation of voltage amplitudes is devised. The latter is rigorously formulated and eight alternative ways to tackle it are proposed. Comparative studies between the proposed versions of the algorithm verify its effectiveness in producing an accurate estimate of the voltage profile, on average in the order of $10^{-3}$ pu close to the exact solution. The proposed algorithm features very favorable computational requirements of approximately a fifth of the time required for an exact solution. Its computational efficiency renders it a solid candidate for hard real-time applications required in the emerging smart grid.
\end{abstract}

Index Terms-- DC power flow, linear systems, overdetermined systems, least-squares method.

\section{INTRODUCTION}

The power flow problem is one of most fundamental problems in power system engineering. It deals with the determination of the voltage profile of a power system, given some generation constraints. Formally the problem can be stated as a system of non-linear equations.

$$
S=\operatorname{diag}(V) \cdot Y^{*} \cdot V^{*}
$$

Numerous well-established solutions exist for the above based on iterative procedures. The Newton-Raphson [4] and the Fast-Decoupled Power Flow methods [5] are perhaps the most well-known and used methods. The DC power flow (DC-PF) is a non-iterative alternative to the above problem [2]. Although the DC power flow provides only an approximate solution to the problem, it still remains very popular, especially in the frame of Location Marginal Pricing (LMP)-base market applications [3]. In its classical formulation, after a sequence of approximations it results in a linear system linking active power injections to bus voltage angles.

A problem with the DC-PF is that it does not give any information on bus voltage magnitudes. Attempts have been made to tackle the problem before [1]. This work deals with the same problem. It provides a rigorous reference on the problem and proposes a set of different solutions. In brief, an estimate of the bus angles is obtained using a classical DC-PF formulation, and then an approximation of the voltage magnitude profile is made, using power balance constraints.

This paper is organized as follows. Section II introduces the reader to the classical DC-PF formulation. Section III extends the latter with bus voltage magnitude estimation. In section IV, results are presented concerning the accuracy and the computational requirements of the proposed method. Finally, conclusions are drawn in section $\mathrm{V}$, and future potential research opportunities are highlighted.

\section{STANDARD DC POWER FLOW FORMULATION}

In the classical textbook formulation of DC PF the following assumptions are made

- No transmission losses

- $\sin \theta_{i k} \approx \theta_{i k}$, supposing small $\theta_{i k}=\theta_{i}-\theta_{k}$

- Flat voltage profile $\left|V_{i}\right| \approx 1$

- $-b \approx 1 / x$, supposing low $r / x$ ratios

Making the assumptions above, (1) becomes

$$
P=H \cdot \theta
$$

Where $P$ are bus active power injections, $\theta$ are bus angles and the coefficient matrix $H$ is real symmetric and nonsingular. Solving for $\theta$ provides the bus angles. For a rigorous mathematical presentation of the above see [9].

Falling back to (1), the known variables now are

- voltage angles $\theta$ at all buses

- voltage magnitudes at PV buses and at the slack bus

- active power injections at PV and PQ buses

- reactive power injections at PQ buses

And the still unknown variables

- $\quad$ voltage magnitudes at PQ buses

- reactive power injections at PV buses

Since all quantities are complex, in total ignoring the slack, there are $2 \cdot\left(n_{P V}+n_{P Q}\right)$ equations and $n_{P V}+n_{P Q}$ unknowns. This means that the system is overdetermined. Next sections deal with the treatment of this system. East

This work is part of the Innovation Program on Energy of EPFL Middle 


\section{PROPOSED FORMULATION}

In the proposed algorithm, the equation is first translated using three transformations. We define:

- $\quad$ the modified admittance matrix $Y_{i k}^{+} \triangleq Y_{i k}^{*} \cdot e^{-j \theta_{k}}$

- $\quad$ the modified apparent power $S_{i}^{+} \triangleq S_{i} \cdot e^{-j \theta_{i}}$

- the bus voltage magnitude is separated into a base value plus a perturbation $V_{i}=\left(V_{i}^{b}+\delta V_{i}\right) \cdot e^{j \theta_{i}}$

Then, (1) can be rewritten as

$$
S_{i}^{+}=\left(V_{i}^{b}+\delta V_{i}\right) \cdot \sum_{k} Y_{i k}^{+} \cdot\left(V_{k}^{b}+\delta V_{k}\right)
$$

For buses $i$ with voltage setpoint $V_{i}^{m}$ (PV and slack) it holds $V_{i}^{b}=V_{i}^{m} \& \delta V_{i}=0$. For PQ buses, it holds $V_{i}^{b} \triangleq 1$. Values $V_{i}^{b}$ can be grouped to a real known vector ${ }_{(n \times 1)} V^{b}$.

For the reference of the reader, the formulas for the real and the imaginary part of the modified admittance and the modified apparent power are as follows

$$
\begin{gathered}
Y_{i k}^{+}=G_{i k}^{+}+B_{i k}^{+} \rightarrow\left\{\begin{array}{c}
G_{i k}^{+} \equiv G_{i k} \cos \theta_{k}+B_{i k} \sin \theta_{k} \\
B_{i k}^{+} \equiv-G_{i k} \sin \theta_{k}-B_{i k} \cos \theta_{k}
\end{array}\right. \\
S_{i}^{+}=P_{i}^{+}+Q_{i}^{+} \rightarrow\left\{\begin{array}{l}
P_{i}^{+} \equiv P_{i} \cos \theta_{i}+Q_{i} \sin \theta_{i} \\
Q_{i}^{+} \equiv Q_{i} \cos \theta_{i}-P_{i} \sin \theta_{i}
\end{array}\right.
\end{gathered}
$$

System (3) is nonlinear. The nonlinearity can be weighed in two ways. They are termed quad-and inv-approximation for the rest of this paper, and they are defined hereunder.

1) Quad-approximation: Expanding (2)

$$
\begin{gathered}
S_{i}^{+}=V_{i}^{b} \cdot \sum_{k} Y_{i k}^{+} \cdot V_{k}^{b}+V_{i}^{b} \cdot \sum_{k} Y_{i k}^{+} \cdot \delta V_{k}+\delta V_{i} \cdot \sum_{k} Y_{i k}^{+} \cdot V_{k}^{b} \\
+\delta V_{i} \cdot \sum_{k} Y_{i k}^{+} \cdot \delta V_{k}
\end{gathered}
$$

The last term contains quadratic perturbation terms $\left(\delta V_{i} \times \delta V_{k}\right)$ and can be neglected under the assumption $\delta V_{j} \cong 0$; so, the system becomes linear.

$$
S_{i}^{+}-V_{i}^{b} \cdot \sum_{k} Y_{i k}^{+} \cdot V_{k}^{b}=V_{i}^{b} \cdot \sum_{k} Y_{i k}^{+} \cdot \delta V_{k}+\delta V_{i} \cdot \sum_{k} Y_{i k}^{+} \cdot V_{k}^{b}
$$

2) Inv-approximation: Considering that $\frac{1}{a+b} \cong a-b$, when $a \cong 1, b \cong 0$. For $V_{i}^{b} \equiv a, \delta V_{i} \equiv b$, (3) becomes

$$
S_{i}^{+} \cdot V_{i}^{b}-\sum_{k} Y_{i k}^{+} \cdot V_{k}^{b}=S_{i}^{+} \cdot \delta V_{i}+\sum_{k} Y_{i k}^{+} \cdot \delta V_{k}
$$

Equations (6) and (7) describe complex linear systems, which can be handled to retrieve the voltage magnitude estimates. Known and unknown variables are summarized in table I. Since the unknown $\delta V$ 's appear only in PQ bus equations, only the latter are retained.

Starting from (6), splitting the sums into slack, PV, and PQ specific ones, and writing in matrix notation, the above becomes

$$
S^{+}-Y^{+} \cdot V^{b}=\left[Y^{+}+\operatorname{diag}\left(Y^{+} \cdot V^{b}\right)\right] \cdot \delta V
$$

TABLE I: KNOWN AND UNKNOWN VARIABLES OF THE TRANSLATED LINEARIZED SYSTEM

\begin{tabular}{ccccc}
\hline \hline & $P_{i}^{+}$ & $Q_{i}^{+}$ & $\delta V_{i}$ & $\theta_{i}$ \\
\hline$n_{S L}$ & $X$ & $X$ & $V$ & $V$ \\
$n_{P V}$ & $X$ & $X$ & $V$ & $V$ \\
$n_{P Q}$ & $V$ & $V$ & $X$ & $V$ \\
\hline \hline
\end{tabular}

Expanding (8) into real and imaginary part yields the system equations for the quad-approximation.

$$
\left\{\begin{array}{l}
P^{+}-G^{+} \cdot V^{b}=\left[G^{+}+\operatorname{diag}\left(G^{+} \cdot V^{b}\right)\right] \cdot \delta V \\
Q^{+}-B^{+} \cdot V^{b}=\left[B^{+}+\operatorname{diag}\left(B^{+} \cdot V^{b}\right)\right] \cdot \delta V
\end{array}\right.
$$

Analogously, starting from (7), the system equations for the inv-approximation are

$$
\left\{\begin{array}{l}
P^{+}-G^{+} \cdot V^{b}=\left[G^{+}+\operatorname{diag}\left(P^{+}\right)\right] \cdot \delta V \\
Q^{+}-B^{+} \cdot V^{b}=\left[B^{+}+\operatorname{diag}\left(Q^{+}\right)\right] \cdot \delta V
\end{array}\right.
$$

\section{B. Dealing with an overdetermined system}

In both systems (9) and (10), there are $2 \cdot n_{P Q}$ equations and $n_{P Q}$ unknowns. There are four different ways to tackle the overdetermined systems. The analysis is done elaborately only for the quad-approximation. In the end of the section analogous results are given for the inv-approximation.

1) P-formulation: If only the modified active power set of equations of (9) is retained, then the resulting linear system is determined, i.e. it has equal number of equations and variables. Subsequently, a unique solution can be attained.

$$
\begin{gathered}
n_{P Q} \times 1 b_{P} \triangleq P^{+}-G^{+} \cdot V^{b} \\
n_{P Q} \times n_{P Q} A_{P} \triangleq G^{+}+\operatorname{diag}\left(G^{+} \cdot V^{b}\right) \\
\delta V=A_{P}^{-1} \cdot b_{P}
\end{gathered}
$$

The above solution ensures, within the limits of the approximations, that the voltage profile begets the required modified active power injection to PQ buses. The latter is through (5) strongly linked to the active power injection to the buses.

2) Q-formulation: Analogously, if only the modified reactive power set of equations of (9) is retained, then the resulting system is again determined

$$
\begin{gathered}
n_{P Q} \times 1 b_{Q} \triangleq Q^{+}-B^{+} \cdot V^{b} \\
n_{P Q} \times n_{P Q} A_{Q} \triangleq B^{+}+\operatorname{diag}\left(B^{+} \cdot V^{b}\right) \\
\delta V=A_{Q}^{-1} \cdot b_{Q}
\end{gathered}
$$

This solution ensures that the voltage profile satisfies the preset modified reactive power injection to PQ buses. The latter is though (5) strongly linked to the reactive power injection to the buses.

For systems with high $x / r$ ratios such as typical transmission level systems, $A_{P}$ is expected to have a higher condition number than $A_{Q}$. This can be explained by the fact 
TABLE II: RESULTING LINEAR SYSTEMS BASED ON DIFFERENT APPROXIMATIONS AND FORMULATIONS

\begin{tabular}{|c|c|c|}
\hline & Quad-approximation & Inv-approximation \\
\hline P-formulation $\delta V=A_{P}^{-1} \cdot b_{P}$ & $\begin{array}{c}b_{P} \triangleq P^{+}-G^{+} \cdot V^{b} \\
A_{P} \triangleq G^{+}+\operatorname{diag}\left(G^{+} \cdot V^{b}\right)\end{array}$ & $\begin{array}{c}b_{P} \triangleq P^{+}-G^{+} \cdot V^{b} \\
A_{P} \triangleq G^{+}+\operatorname{diag}\left(P^{+}\right)\end{array}$ \\
\hline Q-formulation $\delta V=A_{Q}^{-1} \cdot b_{Q}$ & $\begin{array}{c}b_{Q} \triangleq Q^{+}-B^{+} \cdot V^{b} \\
A_{Q} \triangleq B^{+}+\operatorname{diag}\left(B^{+} \cdot V^{b}\right)\end{array}$ & $\begin{array}{c}b_{Q} \triangleq Q^{+}-B^{+} \cdot V^{b} \\
A_{Q} \triangleq B^{+}+\operatorname{diag}\left(Q^{+}\right)\end{array}$ \\
\hline $\begin{array}{c}\text { LSQ-formulation } \\
\min _{\delta V}\left\|A_{L} \cdot \delta V-b_{L}\right\|\end{array}$ & $\begin{aligned} b_{L} & =\left[\begin{array}{ll}b_{Q} & b_{P}\end{array}\right]^{T} \\
A_{L} & =\left[\begin{array}{ll}A_{Q} & A_{P}\end{array}\right]^{T}\end{aligned}$ & $\begin{array}{l}\text { analogous to the quad- } \\
\text { approximation }\end{array}$ \\
\hline $\begin{array}{c}\text { WLSQ-formulation } \\
\min _{\delta V}\left\|W \cdot\left(A_{L} \cdot \delta V-b_{L}\right)\right\|\end{array}$ & $\begin{array}{c}b_{W}=b_{L} \\
A_{W}=A_{L} \\
W=\operatorname{diag}\left(\left[w_{Q} I_{n_{P Q}} \quad w_{P} I_{n_{P Q}}\right]\right)\end{array}$ & $\begin{array}{l}\text { analogous to the quad- } \\
\text { approximation }\end{array}$ \\
\hline
\end{tabular}

that the former relies more heavily on branch conductances $\left|G_{i k}\right| \cong 0$ (through the modified conductances $G_{i k}^{+}$), while the latter relies more on branch susceptances $B_{i k}$ instead (through the modified susceptances $B_{i k}^{+}$). This has been observed in numerical experiments with a variety of systems.

Given the higher degree of coupling between $\mathrm{V}$ and $\mathrm{Q}$ in power systems, the Q-formulation is expected to provide results that are somewhat more precise, compared to the Pformulation. This has been also experimentally observed.

3) LSQ-formulation: In the Least Squares formulation, the linear system matrices are formed as

$$
2 \cdot n_{P Q} \times 1=b_{L}=\left[\begin{array}{l}
b_{Q} \\
b_{P}
\end{array}\right] 2 \cdot n_{P Q} \times n_{P Q} A_{L}=\left[\begin{array}{l}
A_{Q} \\
A_{P}
\end{array}\right]
$$

Obviously, the system is overdetermined. The latter is solved in a least-squares fashion. That is, a solution vector is provided $\delta V \approx A_{L} \backslash b_{L}$, such that the residual is minimized $\min _{\delta V}\left\|A_{L} \cdot \delta V-b_{L}\right\|$. The latter is equivalent to solving the normal equations of the original system $\left(A_{L}^{T} \cdot A_{L}\right) \cdot \delta V=A_{L}^{T}$. $b_{L}$. The solution obtained with this formulation retains information of both (modified) active and reactive power injections, and can be seen as a compromise between the two previous extremes. It is expected that the LSQ-formulation yields solutions "in-between" P- and Q- formulations.

4) WLSQ-formulation: The Weighted Least Squares formulation is the weighted alternative to LSQ. A diagonal weight matrix $W$ is used to assign different importance to equations. Then the system is solve so as to minimize the weighted residual $\min _{\delta V} \| W \cdot\left(A_{L} \cdot \delta V-\right.$ $\left.b_{L}\right) \|$.

The latter is equivalent to solving the weighted normal equations of the original system $\left(A_{L}^{T} \cdot W \cdot A_{L}\right) \cdot \delta V=A_{L}^{T}$. $W \cdot b_{L}$. This formulation can be used to assign different importance to $\mathrm{Q}$ and $\mathrm{P}$ equations. Fixed weight factors can be applied for each of the two, $w_{Q}$ and $w_{P}$ respectively, and $W$ can be formed as $W=\operatorname{diag}\left(w_{Q} \cdot I_{n_{P Q}} \quad w_{P} \cdot I_{n_{P Q}}\right)$.

An automated way of generating $w_{Q}, w_{P}$ is out of the scope of this work, however the following is suggested. The key factor that determines the "importance" of Q- and Pequations is the $r / x$ ratio of the branches of the system. An alternative measure of this is the angle $\varphi_{i k}$ of the complex representation of the elements of the system admittance matrix $Y_{i k}=\left|Y_{i k}\right| \cdot e^{j \cdot \varphi_{i k}}$. Then, the weights can be determined as $w_{P}=\max _{i, k}$ abs $\left(\cos \varphi_{i k}\right)$ and $w_{Q}=1-w_{P}$.

Results similar to the above can be obtained for the Invapproximation. Table II summarizes all eight different resulting systems, categorized according the approximation and formulation of the problem, as defined in previous paragraphs. In any case, the resulting system is solved for $\delta V$, and then $V_{i}=\left(V_{i}^{b}+\delta V_{i}\right) \cdot e^{j \theta_{i}}$ can be employed to get the final voltage magnitude estimates.

Comment: [1] is using the Inv-approximation and the Qformulation, with $D \equiv b_{Q}$ and $L \equiv A_{Q}(D \& L$ are from (7) in [1], and $b_{Q} \& A_{Q}$ are as defined hereinabove).

\section{NUMERICAL RESULTS}

The algorithm presented in the previous section has been implemented in MATLAB, for all approximations and formulations. In this section, all versions will be compared for precision on systems of different size. Additionally, a time profiling will be performed to assess their speed.

\section{A. Precision assessment}

All versions of table II are applied on sample power systems contained in the MATPOWER package [10]. A precise Newton-Raphson (NR) power flow is first run in order to obtain a trusted solution of the steady state.

Different formulations of table II will be referred to using 2-letter subscripts, one for the formulation (P, Q, L for LSQ, and W for WLSQ) and a second for the approximation (Q for Quad, I for Inv); the character X is used as a wildcard.

Precision is understood as "correct $|V|$ prediction". The per-element absolute difference of the magnitude estimate from the "exact" solution is defined as $\Delta V_{m} \triangleq \|\left. V\right|_{X X}-$ $|V|_{N R} \mid$. Two metrics of merit are the average $\overline{\Delta V_{m}}$ and the infnorm $\left\|\Delta V_{m}\right\|_{\infty}$ of $\Delta V_{m}$. Results are presented in table III. 
TABLE III: $\overline{\Delta V_{m}}$ AND $\left\|\Delta V_{m}\right\|_{\infty}$ RESULTS OF DIFFERENT ALGORITHM VERSIONS FOR DIFFERENT POWER SYSTEMS

\begin{tabular}{|c|c|c|c|c|c|c|c|c|c|c|}
\hline \multirow[b]{2}{*}{ Bus } & \multicolumn{2}{|c|}{6 buses } & \multicolumn{2}{|c|}{30 buses } & \multicolumn{2}{|c|}{118 buses } & \multicolumn{2}{|c|}{2383 buses } & \multicolumn{2}{|c|}{3012 buses } \\
\hline & $\overline{\Delta|V|}$ & $\|\Delta|V|\|_{\infty}$ & $\overline{\Delta|V|}$ & $\|\Delta|V|\|_{\infty}$ & $\overline{\Delta|V|}$ & $\|\Delta|V|\|_{\infty}$ & $\overline{\Delta|V|}$ & $\|\Delta|V|\|_{\infty}$ & $\overline{\Delta|V|}$ & $\|\Delta|V|\|_{\infty}$ \\
\hline PQ & 0.0573 & 0.01297 & 0.01297 & 0.02854 & 0.00111 & 0.00848 & 0.03143 & 1.27821 & 0.01297 & 0.02854 \\
\hline QQ & 0.0027 & $7.76 \mathrm{e}-04$ & $7.76 \mathrm{e}-04$ & 0.00417 & $4.36 \mathrm{e}-04$ & 0.00352 & 0.00621 & 0.01311 & $7.76 \mathrm{e}-04$ & 0.00417 \\
\hline LQ & $9.40 \mathrm{e}-04$ & $6.20 \mathrm{e}-04$ & $6.20 \mathrm{e}-04$ & 0.00314 & $2.51 \mathrm{e}-04$ & 0.00291 & 0.00680 & 0.03829 & $6.20 \mathrm{e}-04$ & 0.00314 \\
\hline WQ & 0.00127 & 0.00204 & 0.00204 & 0.00705 & $2.12 \mathrm{e}-04$ & 0.00230 & 0.00712 & 0.04577 & 0.00204 & 0.00705 \\
\hline PI & 0.05183 & 0.01284 & 0.01284 & 0.02843 & 0.00110 & 0.00844 & 0.05416 & 7.24631 & 0.01284 & 0.02843 \\
\hline QI[1] & 0.00287 & 0.00134 & 0.00134 & 0.00515 & $4.36 \mathrm{e}-04$ & 0.00353 & $7.29 \mathrm{e}-04$ & 0.00935 & 0.00134 & 0.00515 \\
\hline LI & $8.41 \mathrm{e}-04$ & $3.57 \mathrm{e}-04$ & $3.57 \mathrm{e}-04$ & 0.00273 & $2.55 \mathrm{e}-04$ & 0.00295 & 0.00106 & 0.03034 & $3.57 \mathrm{e}-04$ & 0.00273 \\
\hline WI & 0.00121 & 0.00168 & 0.00168 & 0.00687 & $2.12 \mathrm{e}-04$ & 0.00234 & 0.00156 & 0.03864 & 0.00168 & 0.00687 \\
\hline
\end{tabular}

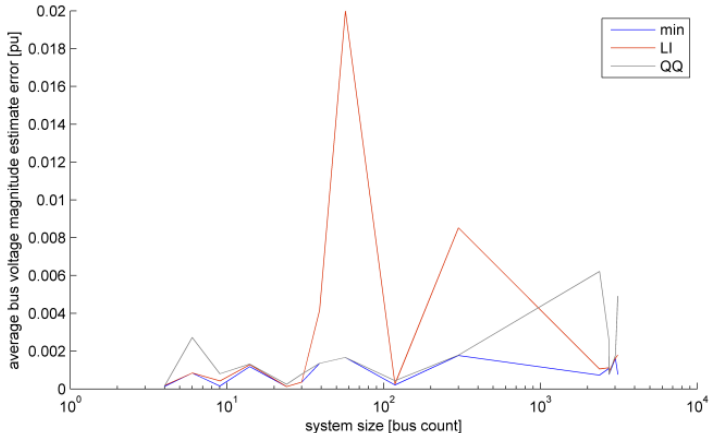

Figure 2. Average bus voltage magnitude estimate error

Empirical examination of the results yields the following general remarks.

- Generally, formulation is more influential than approximation. P-formulations perform poorly; for larger systems the solution collapses to nonsensical estimates. WLSQ-formulations are very sensitive to weight selection.

- The inv-approximation marginally over-performs the quad-approximation. This difference becomes slightly more pronounced in large systems $(>1000$ buses). It is also slightly more pronounced for $\left\|\Delta V_{m}\right\|_{\infty}$ than for $\overline{\Delta V_{m}}$.

- Least-squares versions (LX \& WX) greatly perform better than linear system versions (PX \& QX) in either average or inf-norm metrics. This can be explained by the fact that they have more information at their disposal that the latter. However, this comes at a great computational cost, as it will be shown later in timing results.

- Computationally demanding be it, the LI version generally performs best.

- For linear system versions: QQ performs better for smaller scale systems, while QI performs better for larger scale systems. Aside from precision considerations, linear-system based versions are found to have significant computational benefits.

To sum up, $Q Q$ is the version of the algorithm that is qualified from this work. It will be this version that will be more carefully examined in the rest of this paper. For comparison purposes, results of the LI version will also be quoted.

Fig. 1 and 2 present the average and the maximum bus

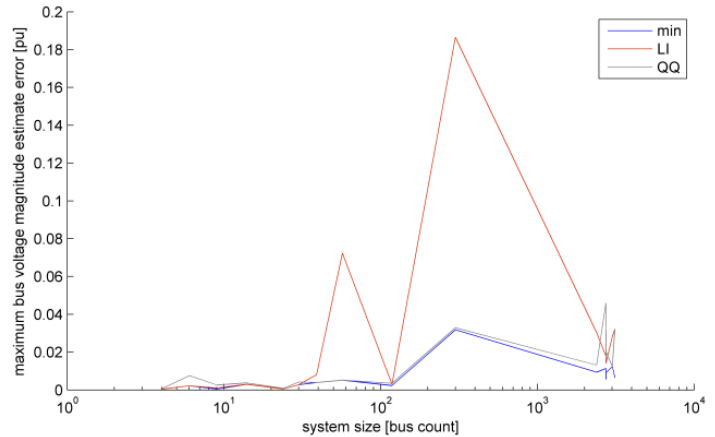

Figure 1. Maximum bus voltage magnitude estimate error

voltage estimate error respectively. Results are given against system size for the minimum achieved error over all versions, for the suggested LSQ based version - LI, and for the suggested linear system based version - QQ. As it can be seen from the figures the quality of the estimate of the algorithms remains quite unaffected by system size.

\section{B. Time profiling}

The two suggested versions of the algorithm, LI and QQ, have been time profiled. For this, an average over multiple runs of the time break-up of the algorithm was done, for a system of 3120 buses. Results of this relative intra-profiling are shown in tables IV and V, for LI and QQ respectively. All computations were done on a typical modern desktop PC (Pentium Core i7 4x2.80 GHz, 8 GB RAM).

\begin{tabular}{lc}
\multicolumn{2}{c}{ TABLE IV: QQ VERSION } \\
TIME PROFILING \\
\hline \hline Operation & Time \\
\hline$\theta$ linear system & $42 \%$ \\
$\mathrm{~V}_{\mathrm{m}}$ linear system & $46 \%$ \\
other & $12 \%$ \\
\hline \hline
\end{tabular}

\begin{tabular}{ll}
\multicolumn{2}{c}{$\begin{array}{c}\text { TABLE V: LI VERSION } \\
\text { TIME PROFILING }\end{array}$} \\
\hline \hline Operation & Time \\
\hline $\begin{array}{l}\text { LSQ problem } \\
\text { other }\end{array}$ & $\begin{array}{l}98 \% \\
2 \%\end{array}$ \\
\hline \hline
\end{tabular}

In the QQ version, it is clearly shown that the PF problem is reduced to the solution of two linear systems, one for the angles and one for the magnitudes. The two, require approximately equal time. All other linear-system-based versions have analogous breakup.

The LI version is dominated by the solution of the LSQ problem. The solution of the linear system of the voltage angles, as per standard DCPF, is included in row "other". All other LSQ-based versions exhibit similar timing characteristics. 
TABLE VI: TIMING COMPARISON BETWEEN NR (BASE), QQ AND LI SOLUTIONS

\begin{tabular}{cccc}
\hline \hline size & $t_{N R}[s]$ & $\frac{t_{Q Q}}{t_{N R}}$ & $\frac{t_{L I}}{t_{N R}}[\mathrm{x} 100]$ \\
\hline 4 & 0.0019 & 0.6729 & 0.0068 \\
6 & 0.0025 & 0.7585 & 0.0076 \\
9 & 0.0032 & 0.6606 & 0.0064 \\
14 & 0.0027 & 0.7689 & 0.0075 \\
24 & 0.0037 & 0.6144 & 0.0060 \\
30 & 0.0036 & 0.6293 & 0.0091 \\
39 & 0.0027 & 0.8329 & 0.0083 \\
57 & 0.0041 & 0.5795 & 0.0071 \\
118 & 0.0056 & 0.5020 & 0.0054 \\
300 & 0.0150 & $\mathbf{0 . 2 9 5 0}$ & 0.0069 \\
2383 & 0.1202 & $\mathbf{0 . 1 5 4 9}$ & 0.3951 \\
2736 & 0.1052 & $\mathbf{0 . 2 1 1 8}$ & 0.7855 \\
2737 & 0.1297 & $\mathbf{0 . 1 7 3 6}$ & 0.6343 \\
2746 & 0.0912 & $\mathbf{0 . 2 2 4 0}$ & 0.7182 \\
3012 & 0.0841 & $\mathbf{0 . 2 8 8 4}$ & 1.1304 \\
\hline
\end{tabular}

In table VI a comparison is presented, between the QQ and LI version of the algorithm, and the standard NR. Time spent for each case is normalized against time spent for NR for the same case.

As it can be seen, the LI version is not really practically applicable, as the solution of the underlying LSQ problem scales badly with system size. Actually, it is orders of magnitude slower than a typical NR method, that can be used instead to solve the system "exactly".

One the other hand, it is well demonstrated that a linear system version of the algorithm can have significant computational benefits compared to a full NR solution; approximately only $1 / 5$ of the time required for large cases.

\section{CONCLUSION}

In this paper an extension to the DC power flow method was presented. In addition to the bus voltage angles of the standard DCPF version, bus voltage magnitudes estimates are provided. For this, the mathematical formulation of the problem was stated, and 8 different ways to tackle it have been proposed. Precision and timing characterization of the methods in question completed the study. The QQ version of the algorithm was qualified as "best", by an empirical study on a plethora of results. A very promising future challenge is to come up with an automated way of selecting the best version of the algorithm for the power system in question.

Applications of the method include real-time securityconstrained planning (SCOPF, SCUC) and market operations (LMP, transmission rights) $[3,7]$. It can also be used as an initial voltage profile guess estimator for iterative methods, in the vein of [1].

There are two key outcomes of this work. First, it transforms the PF problem into two linear systems. This comes in substitution of the usual iterative methods. Second, it drastically reduces computational requirements with a small expense in precision. These two merits can combine in novel scenario-based contingency screening for the smart grid. This could even be implemented in hard real time, using effective computing hardware or software.

The authors would also be very interested to investigate the possibility to translate the current algorithm to a dedicated mixed-signal platform such as the one in [8]. In this way, the inherent parallelism of analog electronics could yield tremendous computational benefits.

\section{REFERENCES}

[1] B. Stott, "Effective starting process for Newton-Raphson load flows," Electrical Engineers, Proceedings of the Institution of, vol. 118, no. 8, pp. 983-987, Aug. 1971.

[2] L. Power, Power System Load Flow Analysis. New York: McGraw-Hill Professional Series, 2004.

[3] B. Stott, J. Jardim, and O. Alsac, "DC Power Flow Revisited," Power System, IEEE Transactions on, vol. 24, no. 3, pp. 1290-1300, Aug.2009.

[4] W. Tinney, and C. Hart, "Power Flow Solution by Newton's Method," Power Apparatus and Systems, IEEE Transaction on, vol. PAS-86, no. 11, pp. 1449-1460, 1967.

[5] B. Stott, and O. Alsac, "Fast Decoupled Load Flow," Power Apparatus and Systems, IEEE Transactions on, vol. PAS-93, no. 3, pp. 859-869, 1974.

[6] P. M. Anderson, and A. A. Fouad, Power system control and stability. Ames: Iowa State University Press, 1977.

[7] M. Shahidehpour, H. Yamin, and Z. Li, Market Operations in Electric Power Systems. New York: John Wiley \& Sons, Inc., 2002.

[8] L. Fabre, G. Lanz, T. Kyriakidis, D. Sallin, I. Nagel, R. Cherkaoui, M. Kayal, "An ultra-high speed emulator dedicated to power system dynamics computation based on a mixed-signal hardware platform," Power Systems, IEEE Transactions on, in press.

[9] R. Kaye, and F. F. Wu, "Analysis of linearized decoupled power flow approximations for steady-state security-assessment," Circuits and Systems, IEEE Transactions on, vol. 31, no. 7, pp. 623-636, Jul 1984.

[10] R. D. Zimmerman, C. E. Murillo-Sánchez, and R. J. Thomas, "MATPOWER: Steady-State Operations, Planning and Analysis Tools for Power Systems Research and Education," Power Systems, IEEE Transactions on, vol. 26, no. 1, pp. 12-19, Feb. 2011. 